Case Report

\title{
Accidental Left Circumflex Artery to Right Lung Fistula in a Suspected Case of Pulmonary Hypertension
}

\author{
Saeed Alipourparsa, ${ }^{1}$ Isa Khaheshi, ${ }^{1}$ Vahid Eslami, \\ Mohammadreza Bozorgmanesh, ${ }^{2}$ and Habib Haybar ${ }^{3}$ \\ ${ }^{1}$ Cardiovascular Research Center, Modarres Hospital, Shahid Beheshti University of Medical Sciences, Tehran, Iran \\ ${ }^{2}$ Prevention of Metabolic Disorders Research Center, Research Institute for Endocrine Sciences, Shahid Beheshti University of \\ Medical Sciences, Tehran, Iran \\ ${ }^{3}$ Cardiovascular Research Center, Ahvaz Jundishapur University of Medical Sciences, Ahvaz, Iran
}

Correspondence should be addressed to Habib Haybar; takamoolsk@yahoo.com

Received 17 April 2014; Revised 29 June 2014; Accepted 29 June 2014; Published 17 July 2014

Academic Editor: Ramazan Akdemir

Copyright (C) 2014 Saeed Alipourparsa et al. This is an open access article distributed under the Creative Commons Attribution License, which permits unrestricted use, distribution, and reproduction in any medium, provided the original work is properly cited.

\begin{abstract}
A 56-year-old woman was referred to the cardiology department of the Shahid Modarres hospital. The patient had a history of pulmonary thromboembolism 20 years ago which had been managed by the inferior vena cava filter and since then the patient has been on warfarin. Her chief complaint was chronic dyspnea on exertion (NYHA class II) from several years ago. Right and left heart catheterization was performed for evaluation of pulmonary artery pressure. We found rich collateral formations between LCX as well as RCA and right pulmonary artery, primarily assumed as multiple fistulas. Among patients who have chronic thromboembolic pulmonary hypertension, systemic collateral supply to the pulmonary parenchyma has been previously reported to occur from both bronchial and/or nonbronchial systemic circulations. Our patient had neither signs of heart failure nor myocardial ischemia and, thus, was a candidate for conservative management. The adenosine pulmonary reactivity test was not performed because of low pulmonary pressure which had been estimated to be high.
\end{abstract}

\section{Introduction}

The case reported herein was a 56-year-old woman with chronic excretion dyspnea (NYHA class II) since 20 years ago when she survived a pulmonary thromboembolism that was managed by the inferior vena cava filter and warfarin. Cardiac examination revealed an irregular rhythm with holosystolic murmur at left lateral sternal border. The physical examination was otherwise unremarkable. On ECG, rhythm was atrial fibrillation $(74 \mathrm{bpm})$ and no significant ST-T changes. Transthoracic echocardiography showed a normal sized LV (LVEDD $=45 \mathrm{~mm}$ ), no regional wall motion abnormality, moderate right ventricle (RV) enlargement with a right ventricular end diastolic dimension of $38 \mathrm{~mm}$, and severe RV systolic dysfunction. There was also tricuspid annulus calcification with a severe tricuspid regurgitation and acceleration time of $89 \mathrm{msec}$ and mean pulmonary artery pressure (PAP) was estimated to be $34 \mathrm{mmHg}$ based on pulmonary valve systolic flow. RV papillary muscles were calcified.

Left heart catheterization and coronary angiography performed via left radial artery revealed normal left and right coronary arteries with rich collateral formations between left circumflex artery and right lung assumed as multiple fistulas (Video 1, Figures 1 and 2; see Supplementary Material available online at http://dx.doi.org/10.1155/2014/427045).

Attempts from both femoral veins for right heart catheterization revealed a cut-off sign at the proximal part of the inferior vena cava (IVC) indicating chronic thrombotic occlusion leading to development of enormous number of collateral veins in both right and left sides, draining the lower limb veins through the hemiazygos (Figure 3 ) and azygos (Figure 4) veins to the right heart (Video 2). Right heart catheterization was performed via the left subclavian vein using a Swan-Ganz catheter. Selective pulmonary angiography revealed proximal occlusion of right pulmonary artery (Video 3, Figure 5), with 


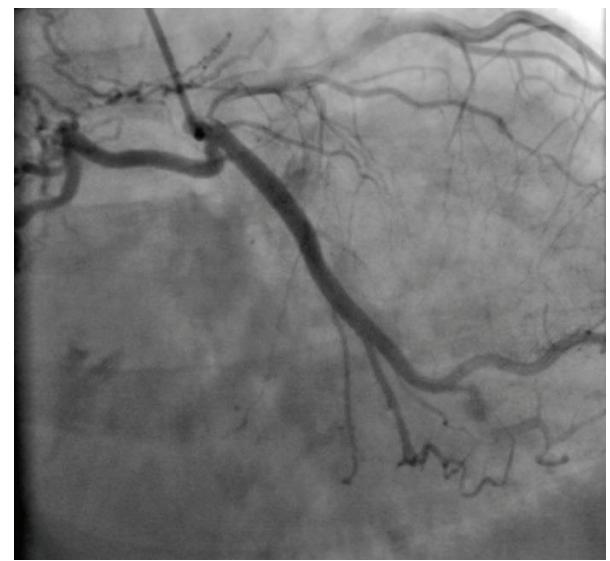

FIGURE 1: Rich collateral formations between left circumflex artery and right lung assumed as multiple fistulas.

RA pressure being $28, \mathrm{RV}$ pressure $42 / 8-12 \mathrm{mmHg}$, $\mathrm{PAP}=$ $48 / 20 \mathrm{mmHg}$, mean PAP $=28 \mathrm{mmHg}$, PCWP $=20 \mathrm{mmHg}$, and $\mathrm{LV}$ pressure $=140 / 10-20 \mathrm{mmHg}$. Flow ratio $\left(Q_{P} / Q_{S}\right)$ for calculation of the size of shunt was as follows:

$$
\frac{Q_{P}}{Q_{S}}=\frac{\mathrm{AoO}_{2}-\mathrm{MVO}_{2}}{\mathrm{PVO}_{2}-\mathrm{PAO}_{2}}
$$

where $Q_{P}$ and $Q_{S}$ represent blood flows in the pulmonary and systemic circulations, respectively. $\mathrm{O}_{2}$ saturations in the right atrium (RA), right ventricle (RV), pulmonary artery (PA), aorta (Ao), and superior and inferior vena cavae (IVC and SVC) were $64 \%, 65 \%, 65 \%, 92 \%, 62 \%$, and $64 \%$, respectively. Mixes venous (MV) $\mathrm{O}_{2}$ saturation was calculated as $62.5 \%$ using this formula:

$$
\begin{aligned}
& \mathrm{MV} \mathrm{O}_{2} \text { saturation } \\
& =\frac{3 * \mathrm{SVCO}_{2} \text { saturation }+\mathrm{IVC} \mathrm{O}_{2} \text { saturation }}{4} .
\end{aligned}
$$

Because there was not any fistula to the left PA and it was the sole accessible vessel in the pulmonary circulation, the flow ratio was calculated to be about 1.

The ratio of the pulmonary vascular resistance (PVR) to the systemic vascular resistance (SVR) was calculated using this formula:

$$
\frac{\mathrm{PVR}}{\mathrm{SVR}}=\frac{\mathrm{PAm}-\mathrm{LAm}}{\mathrm{AOm}-\mathrm{RAm}} \times \frac{Q_{S}}{Q_{P}},
$$

where AOm, LAm, Pam, and RAm are the mean pressures of aorta, left atrium, pulmonary artery, and right atrium, respectively. LAm was estimated using pulmonary capillary wedge pressure (PCWP). PVR/SVR was calculated to be $12 \%$.

\section{Discussion}

During attempts to work up a suspected probable pulmonary hypertension, we found rich collateral formations between LCX as well as RCA and right pulmonary artery, primarily assumed as multiple fistulas. Among patients who have

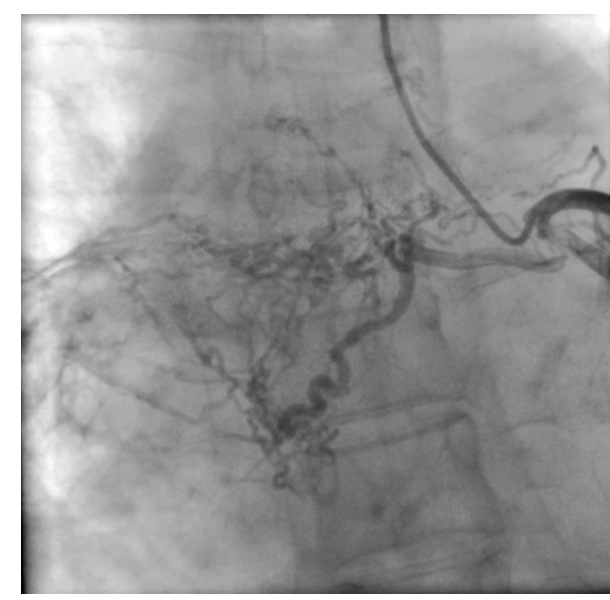

FIGURE 2: Rich collateral formations between left circumflex artery and right lung assumed as multiple fistulas.

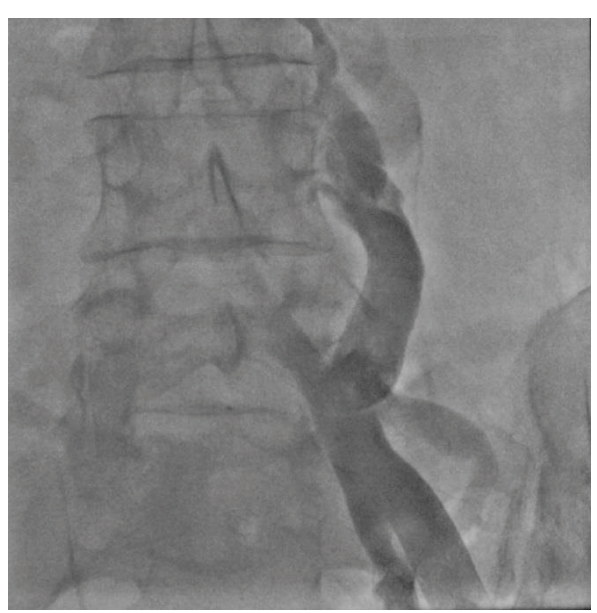

Figure 3: Collateral veins in both right and left sides, draining the lower limb veins through the hemiazygos veins to the right heart.

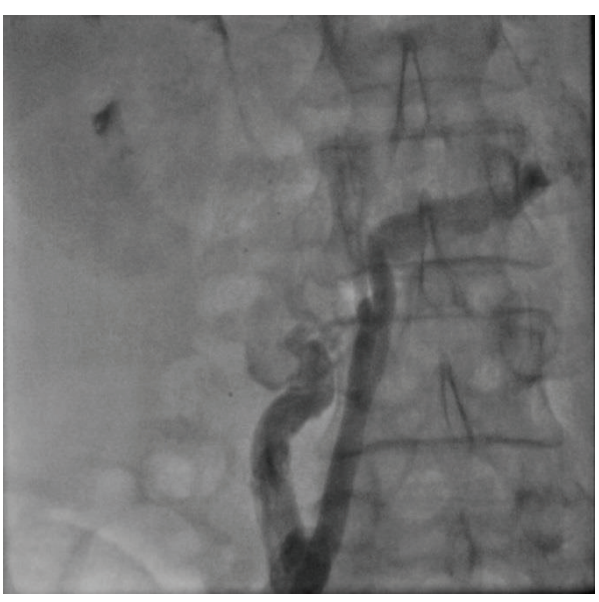

FIGURE 4: Collateral veins in both right and left sides, draining the lower limb veins through the azygos veins to the right heart. 


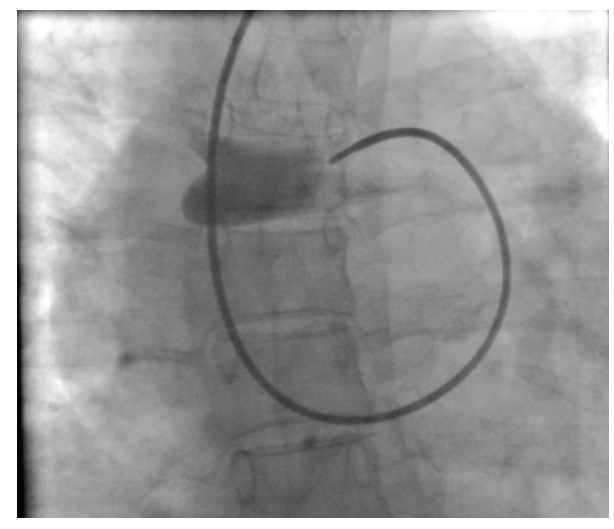

FIGURE 5: Selective pulmonary angiography revealed proximal occlusion of right pulmonary.

chronic thromboembolic pulmonary hypertension, systemic collateral supply to the pulmonary parenchyma has been previously reported to occur from both bronchial and/or nonbronchial systemic circulations [1].

The natural history of coronary artery fistulas is unpredictable. Spontaneous closure secondary to spontaneous thrombosis, although unusual, is a possibility. The management strategy is contentious and most recommendations are based on untrustworthy cases or small retrospective series. Antiplatelet therapy has been advised, particularly in patients with distal coronary artery fistulas and abnormally dilated coronary arteries. Antibiotic prophylaxis against bacterial endocarditis has also been recommended, as bacterial endocarditis is an identified complication. The foremost indications for closure are clinical symptoms of heart failure and myocardial ischemia and sometimes prophylactically in asymptomatic patients with high-flow shunts, to avoid occurrence of symptoms or complications, especially in pediatric population. Surgery and direct epicardial or endocardial ligations were conventionally viewed as the key therapeutic methods for the closure of coronary artery fistulas which is safe and helpful with promising results though treatment of adult asymptomatic patients with nonsignificant shunting is still a matter of debate. Alternatively, catheter-based closure of the fistulous connections as a reasonable nonsurgical option has been recently most popular, with good reported success [2-4].

The adenosine pulmonary reactivity test was not performed because of low pulmonary pressure which had been estimated to be high.

\section{Conflict of Interests}

The authors declare that there is no conflict of interests regarding the publication of this paper.

\section{References}

[1] M. Remy-Jardin, A. Duhamel, V. Deken, N. Bouaziz, P. Dumont, and J. Remy, "Systemic collateral supply in patients with chronic thromboembolic and primary pulmonary hypertension: assessment with multi-detector row helical CT angiography," Radiology, vol. 235, no. 1, pp. 274-281, 2005.

[2] S. Balanescu, G. Sangiorgi, S. Castelvecchio, M. Medda, and L. Inglese, "Coronary artery fistulas: clinical consequences and methods of closure. A literature review," Italian Heart Journal, vol. 2, no. 9, pp. 669-676, 2001.

[3] H. Kamiya, T. Yasuda, H. Nagamine et al., "Surgical treatment of congenital coronary artery fistulas: 27 years' experience and a review of the literature," Journal of Cardiac Surgery, vol. 17, no. 2, pp. 173-177, 2002.

[4] G. G. Hartnell and S. C. Jordan, "Balloon embolisation of a coronary arterial fistula," International Journal of Cardiology, vol. 29, no. 3, pp. 381-383, 1990. 


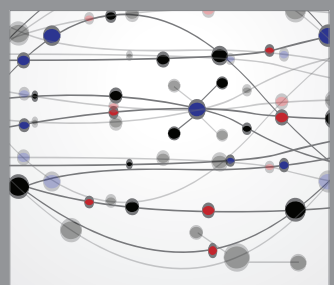

The Scientific World Journal
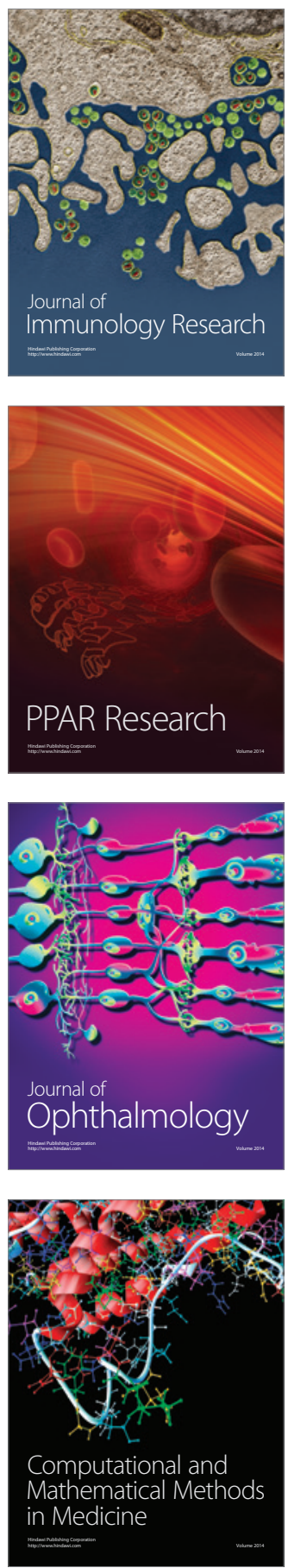

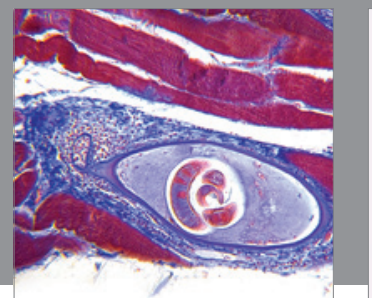

Gastroenterology

Research and Practice
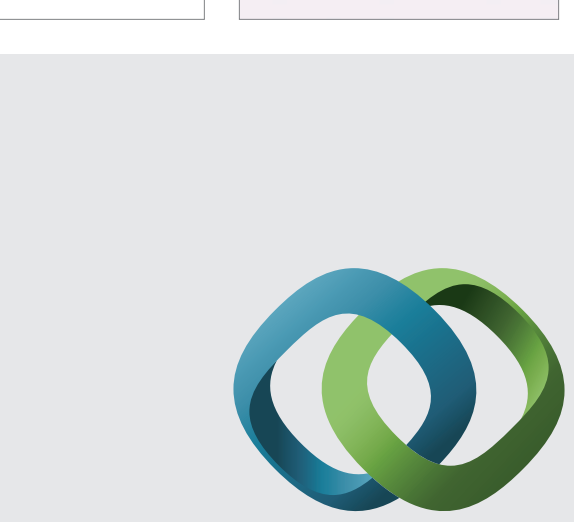

\section{Hindawi}

Submit your manuscripts at

http://www.hindawi.com
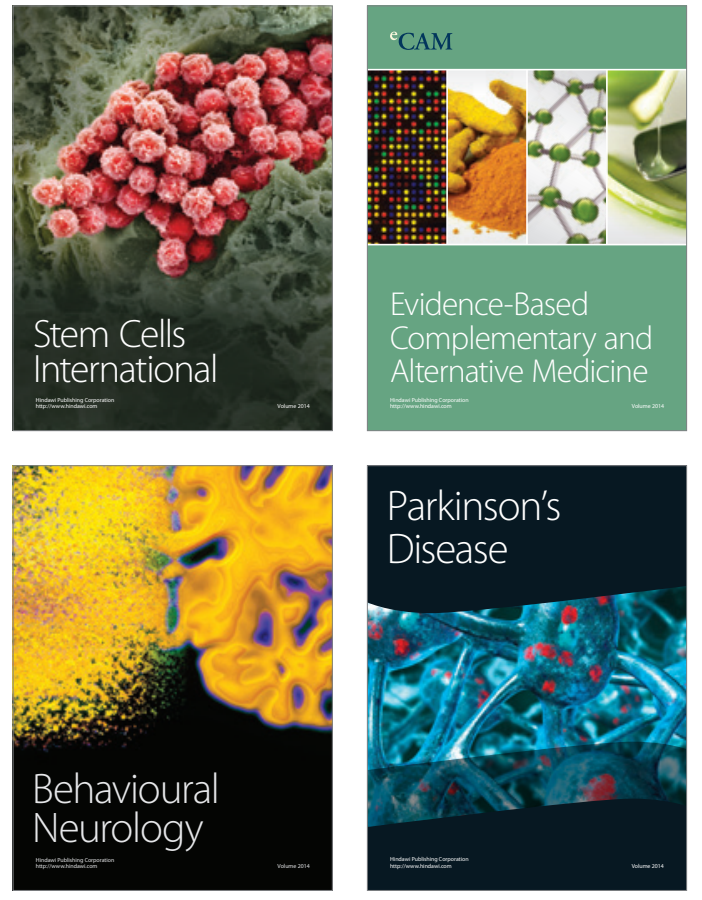
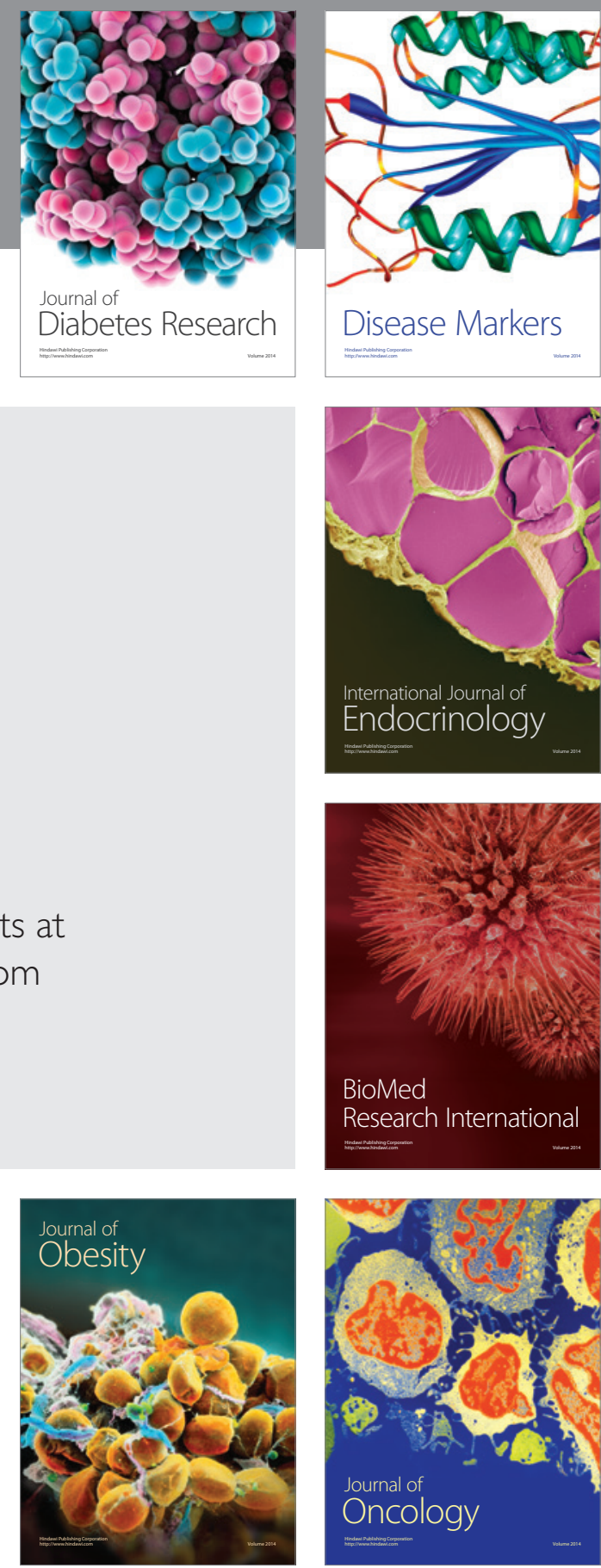

Disease Markers
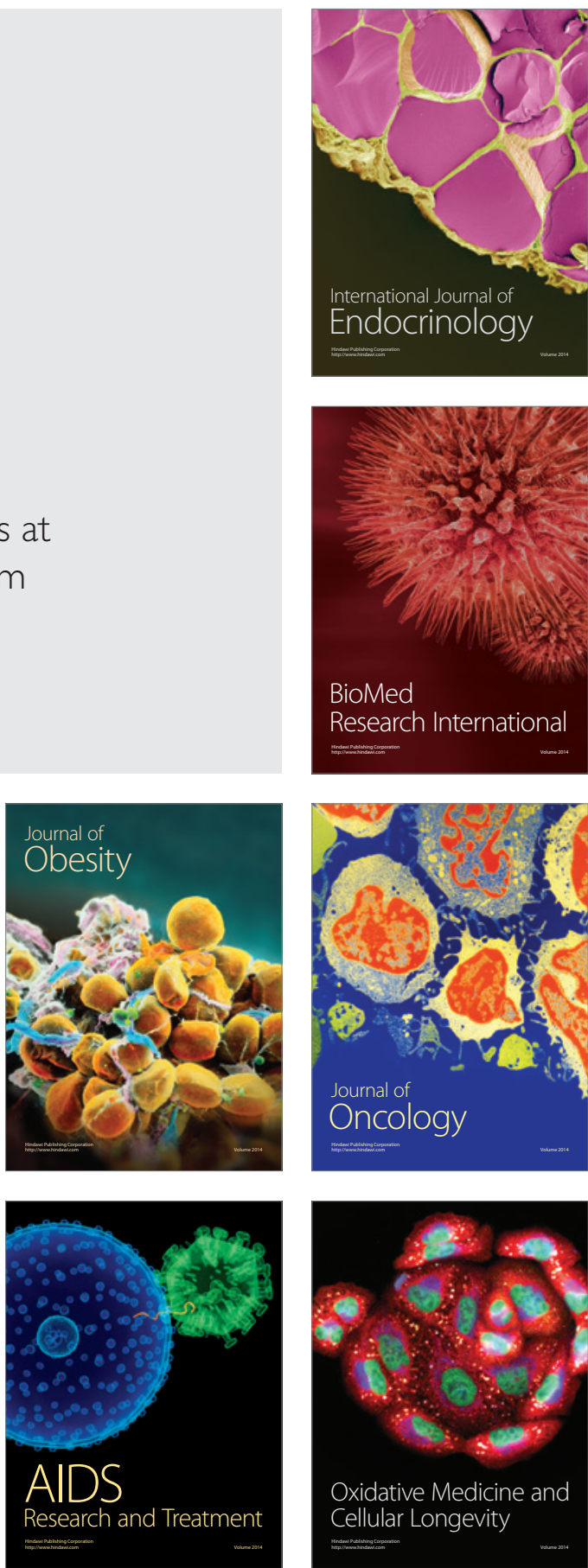